\title{
An investigation of factors affecting the electrospinning of poly (vinyl alcohol)/kefiran composite nanofibers
}

\author{
Hakimeh Ziyadi ${ }^{1} \cdot$ Mitra Baghali $^{2} \cdot$ Mina Bagherianfar $^{2} \cdot$ Fatemeh Mehrali $^{1} \cdot$ Reza Faridi-Majidi $^{3}$
}

Received: 31 October 2020 / Revised: 7 February 2021 / Accepted: 21 February 2021 / Published online: 15 March 2021

(c) The Author(s), under exclusive licence to Springer Nature Switzerland AG 2021

\begin{abstract}
This study aimed to investigate parameters affecting the electrospinning of poly (vinyl alcohol) (PVA)/kefiran composite nanofibers. Accordingly, PVA/kefiran composite nanofibers were produced using the electrospinning of PVA, kefiran blend solutions under various electrospinning parameters (such as applied voltage, nozzle-to-collector distance, and polymer injection rate), and solution parameters (such as the ratio of polymers). PVA and kefiran solutions were prepared in $8 \%$ and $6 \%$ w/w, respectively. Kefiran was blended with PVA solution in different proportions: 70:30, 60:40, 50:50, 40:60, and 30:70. According to the scanning electron microscope (SEM) images, kefiran mixed with PVA in 40:60 ratios produced the best result in nanofiber production. Then, device parameters such as voltage $(12,15,18$, and $20 \mathrm{kV})$, distance $(120,150,170$, and $200 \mathrm{~mm})$, and polymer injection rates $(1,1.5,2$, and $2.5 \mathrm{~mL} / \mathrm{h})$ were changed. The investigation of SEM images showed that the optimal condition for the fabrication of nanofibers was $18 \mathrm{kV}, 200 \mathrm{~mm}$, and $1 \mathrm{~mL} / \mathrm{h}$. The nanofibers produced in the optimal condition were uniform without knots or adhesion in a small diameter. It was also found that concentration can be regarded as the most effective parameter affecting the diameter of nanofibers. Moreover, the transmission electron microscopy (TEM) image proved that phase separation did not occur between the two polymers.
\end{abstract}

Keywords Poly(vinyl alcohol $) \cdot$ Kefiran $\cdot$ Electrospinning $\cdot$ Nanofiber $\cdot$ Optimization

\section{Introduction}

The electrospinning technique has attracted considerable attention in academic and industrial societies due to its easy and efficient capacity to produce nanofibers [1-3]. Obtained nanofibers from this technique have different uses in medical, tissue engineering, drug delivery, filtration, preservative coatings, catalytic reactions, sensors, and lithium-ion batteries [4-12]. Different types of polymers are electrospinned to nanofibers, but the replacement of raw materials of petrochemical products with agricultural

Hakimeh Ziyadi

behnazziyadi@yahoo.com

1 Department of Organic Chemistry, Faculty of Pharmaceutical Chemistry, Tehran Medical Sciences, Islamic Azad University, Tehran, Iran

2 Active Pharmaceutical Ingredients Research Center, Tehran Medical Sciences, Islamic Azad University, Tehran, Iran

3 Department of Medical Nanotechnology, School of Advanced Medical Technologies, Tehran University of Medical Sciences (TUMS), Tehran, Iran and microbial products is highly desirable due to health and environmental issues. Many biopolymers, such as cellulose, chitosan, and collagen, have been electrospinned successfully [13-16]. Many biopolymers have been dissolved and electrospuninned with harmful solvents such as hydrochloric acid and chloroform. As a result, the presence of harmless solvents that are in good agreement with biopolymers is required for fiber production. Hence, in line with the goals of green chemistry, we decided to use poly. (vinyl alcohol) (PVA) and kefiran mixture as soluble polymers in water for the first time.

Kefir is edible acid-alcohol fermented milk that can be easily produced from mixing kefir grains and yeasts with fresh milk at room temperature. The antimicrobial composition and anti-inflammatory action of kefir can make it a good inhibitor of the "cytokine storm" and thus protect us from the COVID-19 disease [17]; hence, further modification of kefir and the fabrication of different materials and composites can be important to the development of a suitable efficacious antiviral drug to treat infected patients [18]. Kefir grain consists of water-soluble exopolysaccharide kefiran, which consists of equivalent amounts of galactose 
and glucose [19]. Kefiran is a green and pharmaceutical biopolymer which has an immune system modification with antibacterial, antifungal, antitumor, and anti-inflammatory activities [20, 21]. Kefiran nanofibers have been previously electrospinned singly, and the electrospinnability of different solutions of kefiran has been discussed [22]. But it seems that due to the high solubility of produced kefiran nanofibers in water and their frangibility, it is essential to combine it with other polymers and prepare suitable composites.

The studies thus far have proved that biodegradable polymer-based composite structures are best to be used in food industries and medical applications [23]. Composite polymer nanofibers have attracted a lot of interest for use in various biomedical and food packaging applications in the last decade [24, 25]. Owing to the abundance and low cost of polysaccharides, the composite nanofibers, made out of these natural polymers (e.g., cellulose, chitosan) together with biodegradable synthetic polymers (e.g., PLA, PVA), are suitable to be used in food packaging materials [26, 27]. For instance, Jenab et al. fabricated kefiran/polyethylene oxide nanofibers for food packaging [28]; however, to the best of our knowledge, other composite nanofibers of kefiran have not been fabricated so far.

PVA is one of the first artificial test polymers used to make artificial cartilage, implants, artificial cornea, wound healing, artificial skin, and controlled release drugs [29-31]. This polymer is a biocompatible, biodegradable, and nontoxic polymer; it is a carrier for medication and has a good chemical and biological resistance, high tensile strength, hydrophilicity, and high ability to form fibers [9]. Different composite nanofibers of PVA with natural structure such as gum [32], chitosan [33], cellulose [34], collagen [35], and keratin [36] have been fabricated by the electrospinning method in order to improve mechanical properties, electrospinability, tensile strength, and chemical resistance of biopolymers [37]. Nano-structures such as nano-films from PVA and kefiran polymer mixtures have been also synthesized [38]; yet, a study on the preparation of kefiran/PVA composite nanofibers was conducted by our research group for the first time promising to introduce novel antibacterial and antiviral coating and films based on kefiran nanofibers for application in the medicine, drug, and food industries especially milk packaging industry [39]. Following our previous work, we planned to examine the effect of different parameters on the fabrication and morphology of kefiran/ PVA composite nanofibers. Various parameters are effective in converting polymer to nanofibers via the electrospinning method, including (1) solution parameters such as concentration, molecular weight, viscosity, elasticity, conductivity, and surface tension; (2) device parameters (variables) such as high voltage, the distance between the tip of the nozzle, injection rate of polymer, and drum speed; and (3) environmental parameters such as temperature, humidity, and air velocity in the device [40, 41]. Herein, different effective parameters in the production of nanofibers, three parameters of the process (voltage, distance, injection rate), and the mixing ratio of two polymers as solution parameters were investigated. The aim of verifying parameters is to produce the finest nanofibers possible without knot or adhesion and achieve the lowest diameter of nanofibers.

\section{Experimental}

\subsection{Activation of kefir grains}

Kefir grains were bought from a household shop in Tehran, Iran. One hundred grams of kefir grains was cultured in $800 \mathrm{~mL}$ skimmed milk in a glass container with a closed sealed door at room temperature in the dark for $24 \mathrm{~h}$. Afterwards, the milk which was fermented by kefir was separated from grains. The grains were washed in water and cultured in fresh milk again. This procedure was done several times for 15 days in order to activate grains.

\subsection{Extraction, purification, and isolation of kefiran}

A weighed amount of cultured kefir grains was treated in boiling distilled water with the weight ratio of $1: 10$ for 30 min with continuous stirring. The obtained mixture was centrifuged (at $10,000 \mathrm{rpm}$ ) for $20 \mathrm{~min}$ at $20{ }^{\circ} \mathrm{C}$. The polysaccharide in the supernatant was precipitated by adding two volumes of cold ethanol $(96 \%$, Merck) and left overnight at $20^{\circ} \mathrm{C}$. After $24 \mathrm{~h}$, the obtained mixture was centrifuged for $20 \mathrm{~min}$ at $4^{\circ} \mathrm{C}$. Thereafter, the pellets were dissolved in hot water and the precipitation process was repeated twice. Finally, the precipitate was heated at $60{ }^{\circ} \mathrm{C}$ in the oven for $48 \mathrm{~h}$ to obtain dried kefiran.

\subsection{Preparation of kefiran solution}

Dried kefiran polymer $(0.15 \mathrm{~g})$ was added to $5 \mathrm{~mL}$ boiling distilled water and was stirred by a magnetic mixer for $3 \mathrm{~h}$ at $90{ }^{\circ} \mathrm{C}$ in order to achieve a homogeneous solution of kefiran. Then, $3 \% \mathrm{w} / \mathrm{w}$ of kefiran solution was obtained. The other concentrations of kefiran solution were prepared in the same method.

\subsection{Preparation of blended PVA/kefiran polymeric solution}

PVA powder $\left(\mathrm{Mw}=88,000 \mathrm{~g} \mathrm{~mol}^{-1}, 88 \%\right.$ hydrolyzed $)$ was purchased from Sigma. Eight grams of the PVA powder was dissolved in $100 \mathrm{~mL}$ distilled water $(8 \% \mathrm{w} / \mathrm{w})$ at $60^{\circ} \mathrm{C}$ and was stirred to obtain a homogeneous solution. PVA solution 
and kefiran solutions (in different concentration) were mixed together slowly using a magnetic stirrer at $100{ }^{\circ} \mathrm{C}$ for $3 \mathrm{~h}$ in different proportions. Also, $4 \%$ w/w Tween 80 surfactant was added to these mixtures in order to gain a homogenous solution, to decrease the surface tension of obtained mixtures, to have a continuous electrospinning process, and to create fine and smooth fibers.

\subsection{Electrospinning process}

The electrospinning process was carried out using Electroris (Fanavaran Nano Meghyas Ltd., Co., Tehran, Iran). This machine includes controllable high-voltage power supply with output capability $0-35 \mathrm{kV}$ direct current (DC) and 0-2 $\mathrm{mA}$ to charge the polymer solution across the needle, a syringe pump with a precision of $0.01 \mu \mathrm{L} / \mathrm{h}$ injection rate, and a stainless-steel collector with a rotation speed up to 3500 rpm wrapped by aluminum foil during the electrospinning process. A typical syringe $(5 \mathrm{~mL})$ was used to inject the polymer solution, and the 18-gauge needle was used as a nozzle. Different electrospinning conditions were conducted by changing the main parameters including polymer/kefiran proportion, applied voltage, nozzle-to-collector distance, and injection rate.

\subsection{Characterization}

The nanofibers obtained on the aluminum foil mounted on the rotating drum were used for further analysis. Foil ( $3 \mathrm{~mm} \times 3 \mathrm{~mm}$ pieces) was cut and coated with gold (sputter coater Bal-Tech, 005 SCD, USA). The pieces were sandwiched with carbon adhesive on a sample holder using a scanning electron microscope (Philips XL30 model, USA) at an accelerating voltage of $25 \mathrm{kV}$. Microstructure Measurement Software was used to measure the diameters of 20 nanofibers of each SEM image. The obtained information was entered into the 2010 Microsoft Office Excel and Origin program to draw the distribution diagram of nanofibers. An optical microscopy image of solution was obtained by an Optical microscope (Labomed Lx500, USA). The homogeneity of the structure was characterized by transmission electron microscopy (TEM, EM10C, Zeiss). The samples for TEM observation were prepared in the following manner. First, nanofibers were collected on a glass slide ensuring that fibers were fluffily placed on the glass slide instead of winding firmly. Second, the carbon-coated copper grids were gently held with delicate tweezers on the collector to directly obtain a thin layer of fibers while rubbing back and forth on it.
Table 1 The effect of different parameters on diameters of nanofibers extracted from SEM images

\begin{tabular}{lllllll}
\hline Sample & $\begin{array}{l}\text { SEM } \\
\text { image } \\
\text { (Fig.) }\end{array}$ & Voltage (k) & $\begin{array}{l}\text { Injection } \\
\text { rate }(\mathrm{mL} / \mathrm{h})\end{array}$ & $\begin{array}{l}\text { Kefiran/PVA pro- } \\
\text { portion } \% \text { w/w) }\end{array}$ & $\begin{array}{l}\text { Nozzle to drum } \\
\text { distance }(\mathrm{mm})\end{array}$ & $\begin{array}{l}\text { Nanofib- } \\
\text { ers diameter } \\
\text { (nm } \pm \text { SD) }\end{array}$ \\
\hline a & 1. a. & 15 & 2 & $30 / 70$ & 150 & $475 \pm 45$ \\
$\mathrm{~b}$ & 1. b. & 15 & 2 & $40 / 60$ & 150 & $500 \pm 55$ \\
$\mathrm{c}$ & 1.c. & 15 & 2 & $50 / 50$ & 150 & $538 \pm 58$ \\
$\mathrm{~d}$ & 1. d. & 15 & 2 & $60 / 40$ & 150 & $653 \pm 42$ \\
$\mathrm{e}$ & 1. e. & 15 & 2 & $70 / 30$ & 150 & $766 \pm 43$ \\
$\mathrm{f}$ & 2. a. & 12 & 2 & $40 / 60$ & 150 & $646 \pm 49$ \\
$\mathrm{~g}$ & 2. b. & 15 & 2 & $40 / 60$ & 150 & $500 \pm 55$ \\
$\mathrm{~h}$ & 2. c. & 18 & 2 & $40 / 60$ & 150 & $482 \pm 57$ \\
$\mathrm{i}$ & 2. d. & 20 & 2 & $40 / 60$ & 150 & $452 \pm 44$ \\
$\mathrm{j}$ & 3. a. & 18 & 2 & $40 / 60$ & 120 & $488 \pm 45$ \\
$\mathrm{k}$ & 3. b. & 18 & 2 & $40 / 60$ & 150 & $482 \pm 57$ \\
$\mathrm{l}$ & 3. c. & 18 & 2 & $40 / 60$ & 170 & $398 \pm 42$ \\
$\mathrm{~m}$ & 3. d. & 18 & 2 & $40 / 60$ & 200 & $385 \pm 56$ \\
$\mathrm{n}$ & 4. a. & 18 & 1 & $40 / 60$ & 200 & $305 \pm 35$ \\
o & 4. b. & 18 & 1.5 & $40 / 60$ & 200 & $343 \pm 29$ \\
$\mathrm{p}$ & 4. c. & 18 & 2 & $40 / 60$ & 200 & $385 \pm 56$ \\
$\mathrm{q}$ & 4. d. & 18 & 2.5 & $40 / 60$ & 200 & $12 \pm 459$ \\
\hline
\end{tabular}

$S D$ standard deviations 


\section{Result and discussion}

\subsection{Electrospinning of kefiran/PVA nanofiber}

To prepare an electrospinning solution, a PVA solution $(8 \% \mathrm{w} / \mathrm{w})$ was prepared. For this purpose, the polymer was dissolved in water as a green solvent along with stirring in high temperature to obtain a uniform and homogenous solution. According to our previous study, $8 \% \mathrm{w} / \mathrm{w}$ of PVA in water is the most appropriate concentration for PVA electrospinning [39]. The electrospinning of this polymer solution was done successfully in electrospinning conditions as follows: nozzle-to-collector distance of $120 \mathrm{~mm}$, voltage of $12 \mathrm{kV}$, and injection rate of $1 \mathrm{~mL} / \mathrm{h}$. The obtained nanofibers were continuous and uniform with the average diameter of $254 \mathrm{~nm}$. The sole purpose of the PVA electrospinning was to study the effect of kefiran addition to the morphology and mean diameter of PVA nanofibers. Faridi et al. electrospinned kefiran singly and studied the effects of parameters on the electrospinning of kefiran [22]. Their study showed that by increasing the kefiran concentration up to $4 \% \mathrm{wt}$, beadless and uniform fibers were formed, so in order to produce a polymer mixture with a suitable concentration for electrospinning, 8\% PVA.

solution and 6\% kefiran solutions were provided. At first, these polymers were mixed with a weight ratio of 50:50 under stirring to obtain a uniform solution. The resulting blends were tested in an Electroris device for spinning in the reference condition that PVA electrospinned $(D=120 \mathrm{~mm}$, $V=12 \mathrm{kV}, \mathrm{IR}=1 \mathrm{~mL} / \mathrm{h}$ ). In these conditions, no electrospinning jet was formed and nanofibers did not appear, although after a few minutes, the phase separation between the polymers was observed in the syringe. For this reason, to reduce the surface tension of the resulting mixture and to have a homogenous solution, 4\% wt Tween 80 surfactant was added to the PVA/kefiran solution. After mixing the polymers with the surfactant, the nanofibers were formed, but the polymer jet was not continuous. Rising the temperature to $40{ }^{\circ} \mathrm{C}$ improved the nanofibers production. To evaluate the effect of different parameters such as the mixing ratio of two polymers, voltage, nozzle-to-collector distance, and the rate of polymer injection on the nanofibers morphology and diameters, experiments were designed on at a time. For this purpose, one of parameters was changed in five different levels and other parameters were fixed in the reference condition. Table 1 (samples a-q) summarizes experiments conducted in this study to investigate the electrospinnability of PVA/kefiran solution in different conditions. All experiments produced nanofibers and were characterized with SEM analysis. The SEM image of nanofibers was opened in Microstructure Measurement Software. Then, the diameters of about 20 nanofibers were measured one by one in each
Fig. 1 SEM image of kefiran/PVA nanofiber in different proportions: a $30 / 70$, b $40 / 60$, c 50/50, d 60/40, and e $70 / 30 \%$ W/W in $15 \mathrm{kV}$ voltage, $2 \mathrm{~mL} / \mathrm{h}$ injection rate, and $150 \mathrm{~mm}$ nozzle-to-drum distance

image. The produced data were transferred to Origin software, and the histogram of diameter distribution was drawn. The mean nanofiber diameters $(\mathrm{nm})$ and standard deviations (SD) that were extracted from SEM images using the abovementioned software are presented in Table 1. Optimal conditions were determined based on the morphology and diameter of nanofibers in SEM images. The best nanofibers have a fine and smooth morphology and lowest diameter with narrow distribution and without any adhesion or knots.

\subsection{Effect of kefiran/PVA proportion}

In order to study the effect of kefiran proportion on nanofiber morphology, $6 \%$ concentration of kefiran was prepared in water and mixed with $8 \%$ PVA in different proportions (30/70, 40/60, 50/50, 60/40, 70/30\% w/w kefi$\mathrm{ran} / \mathrm{PVA})$. The obtained solutions were introduced into the syringe and placed in an electrospinning apparatus. Electrospinning conditions in all experiments (Table 1 (a-e)) were as follows: the nozzle-to-collector distance of $150 \mathrm{~mm}$, the voltage of $15 \mathrm{kV}$, and the injection rate of $2 \mathrm{~mL} / \mathrm{h}$.

As can be seen in Fig. 1, nanofibers were produced successfully in different proportions of PVA/kefiran. Nanofiber diameter increased with the increase of kefiran proportion from 475 to $766 \mathrm{~nm}$ (Table 1 (a-e)). When the PVA/kefiran proportion was 70/30, the thickest nanofibers with some knots were produced. While in 60/40, adhesions between nanofibers were seen clearly in a large amount. The proportion of 50/50 and 30/70 also had some adhesions. Therefore, we decided to use $40 / 60$ proportion as optimum kefiran/PVA proportion in the subsequent experiments.

\subsection{Effect of applied voltage on nanofiber diameter}

Considering the impact of the voltage on the diameter of the nanofibers, it was decided to investigate the effect of voltage on the diameter of the kefiran/PVA composite nanofibers. To do so, different amounts of voltages $(12,15,18$, and $20 \mathrm{kV})$ were applied between nozzles and collectors. Figure 2 a-d show the SEM images of these nanofibers respectively, and the mean diameter of nanofibers are presented in Table $1(\mathrm{f}-\mathrm{i})$. Figure 2 proves that the diameter of nanofibers decreased as the voltage increased. The lowest diameter of kefiran/PVA composite nanofibers was related to $20 \mathrm{kV}$, but these nanofibers 

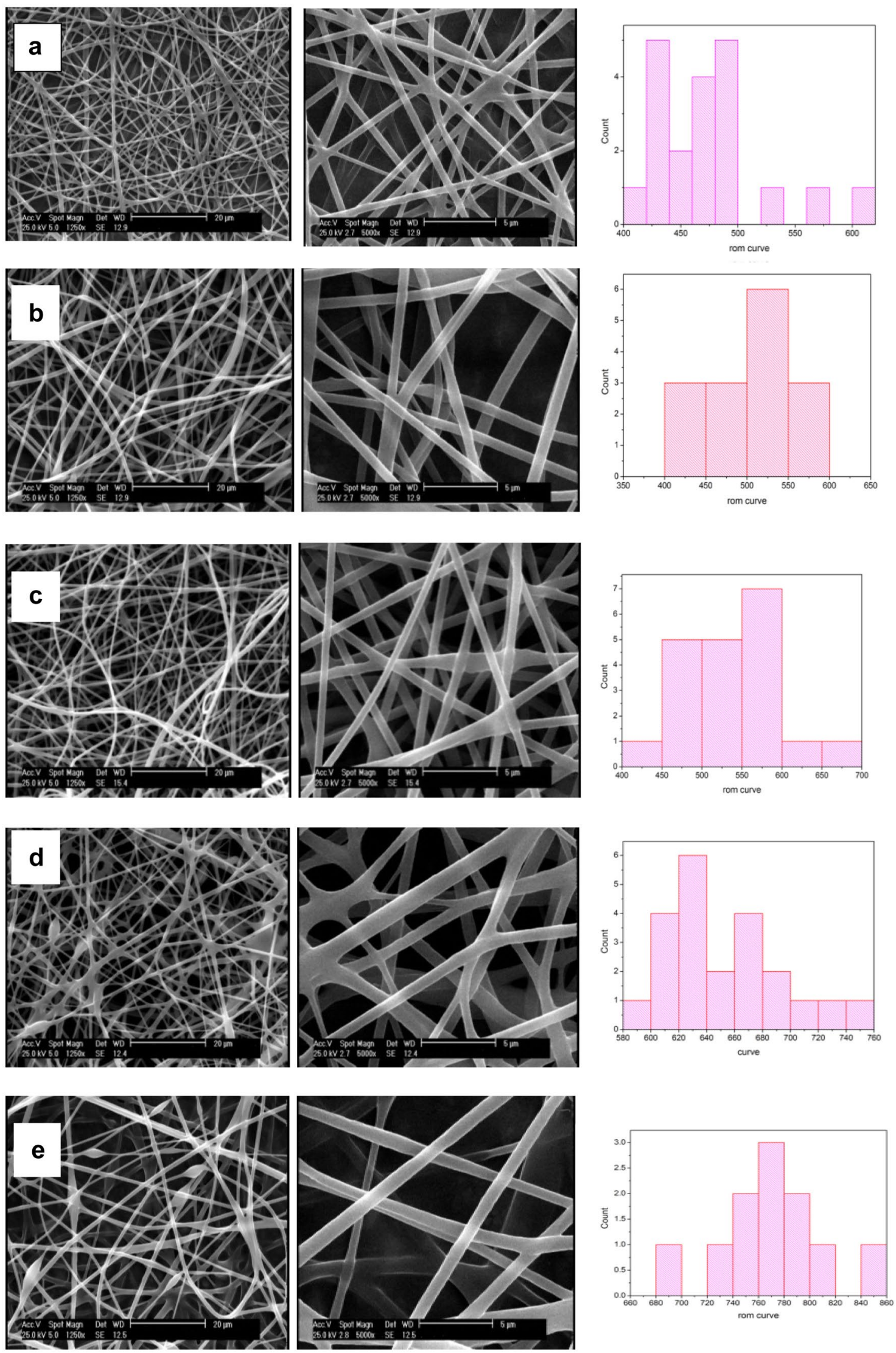

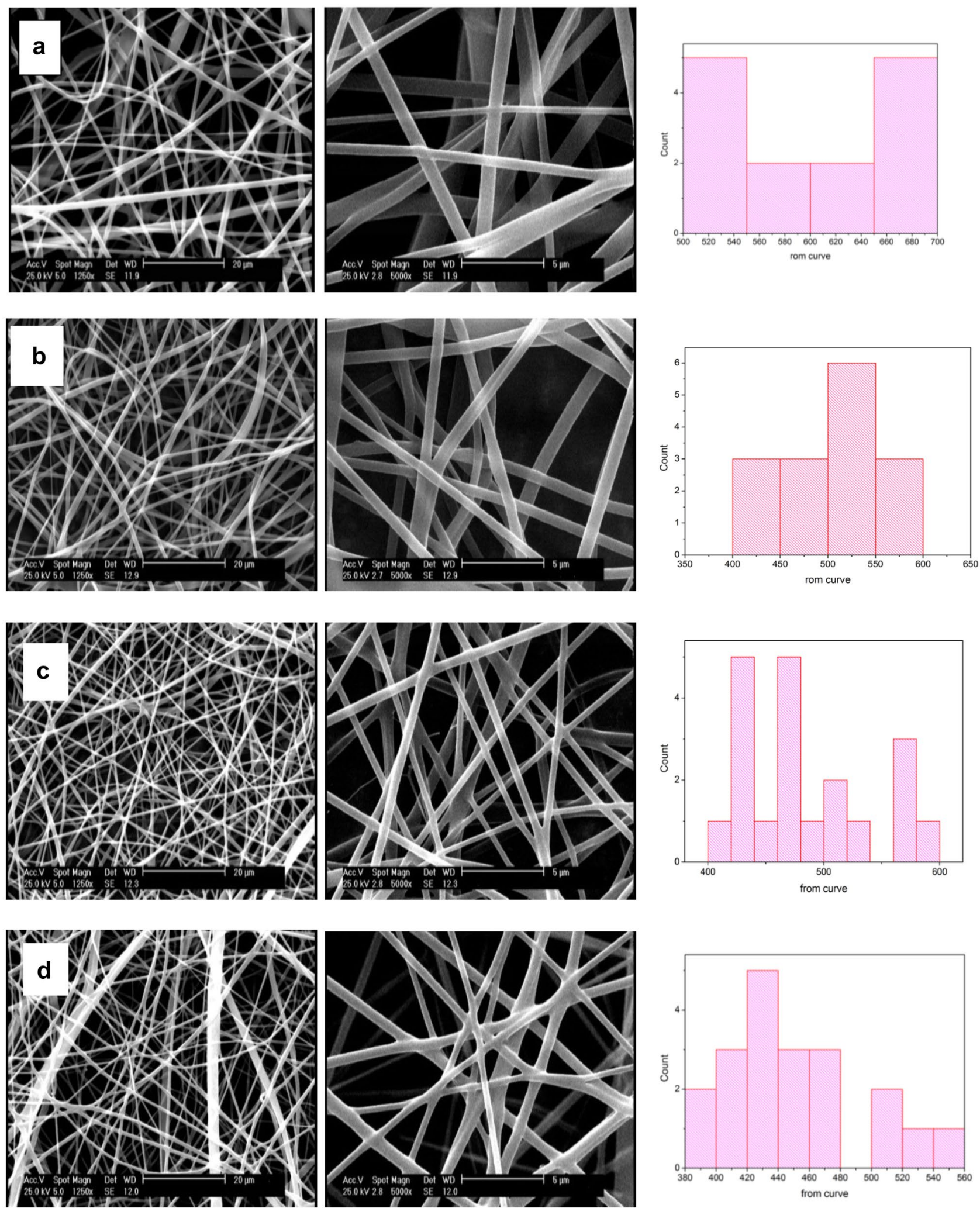

Fig. 2 SEM image of kefiran/PVA nanofiber in different voltages: a 12, b 15, c 18, and d $20 \mathrm{kV}$ in 40/60\% w/w, $2 \mathrm{~mL} / \mathrm{h}$ injection rate, and $150 \mathrm{~mm}$ nozzle-to-drum distance 

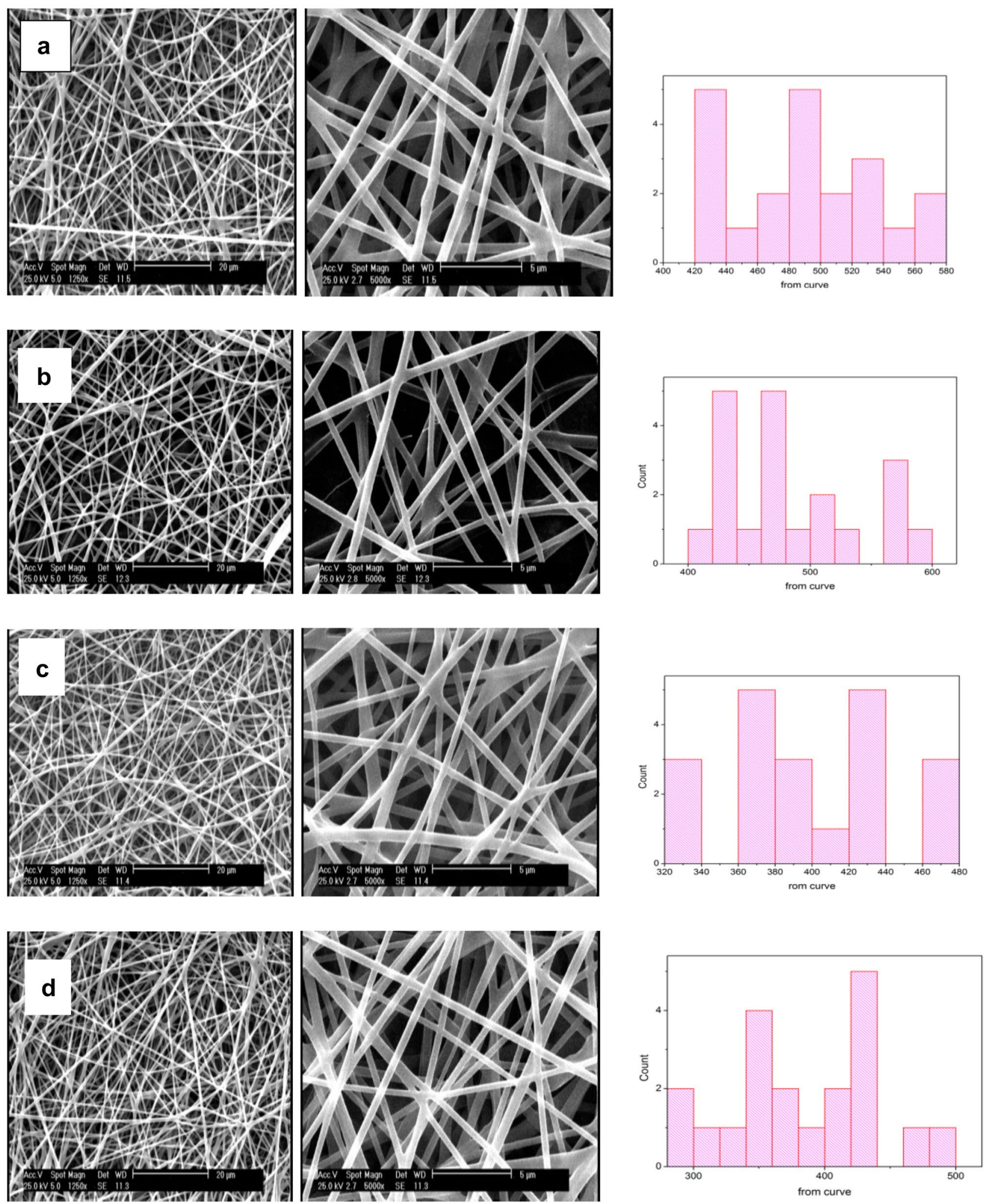

Fig. 3 SEM image of kefiran/PVA nanofiber in different nozzle-to-drum distance: a 120, b 150, c 170, and d 200 mm in 40/60\% w/w, $2 \mathrm{~mL} / \mathrm{h}$ injection rate, and $18 \mathrm{kV}$ voltage 

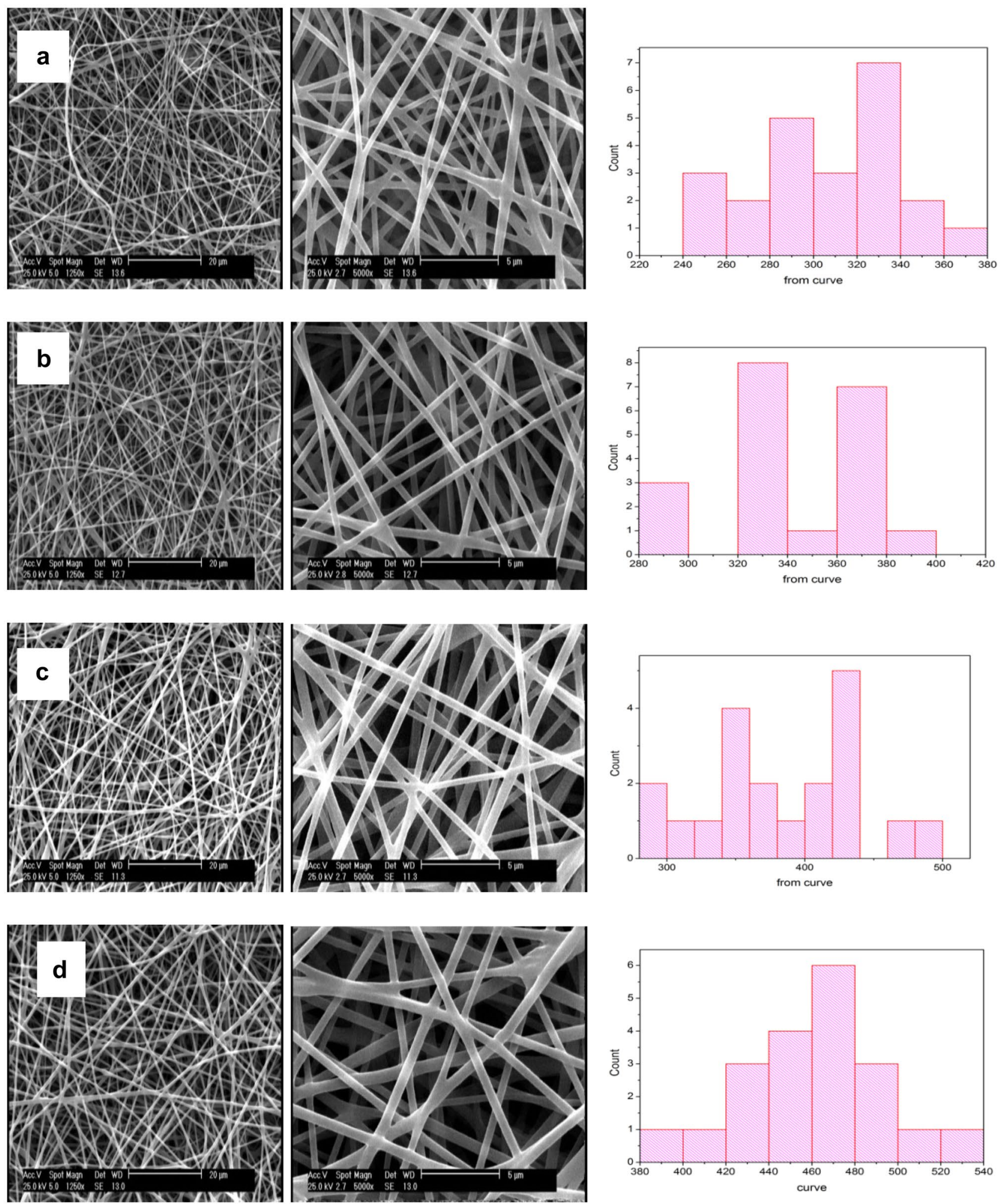

Fig. 4 SEM image of kefiran/PVA nanofiber in different injection rates: a 1, b 1.5, c 2, and d $2.5 \mathrm{~mm}$ in 40/60\% w/w, $200 \mathrm{~mm}$ distance, and $18 \mathrm{kV}$ voltage 

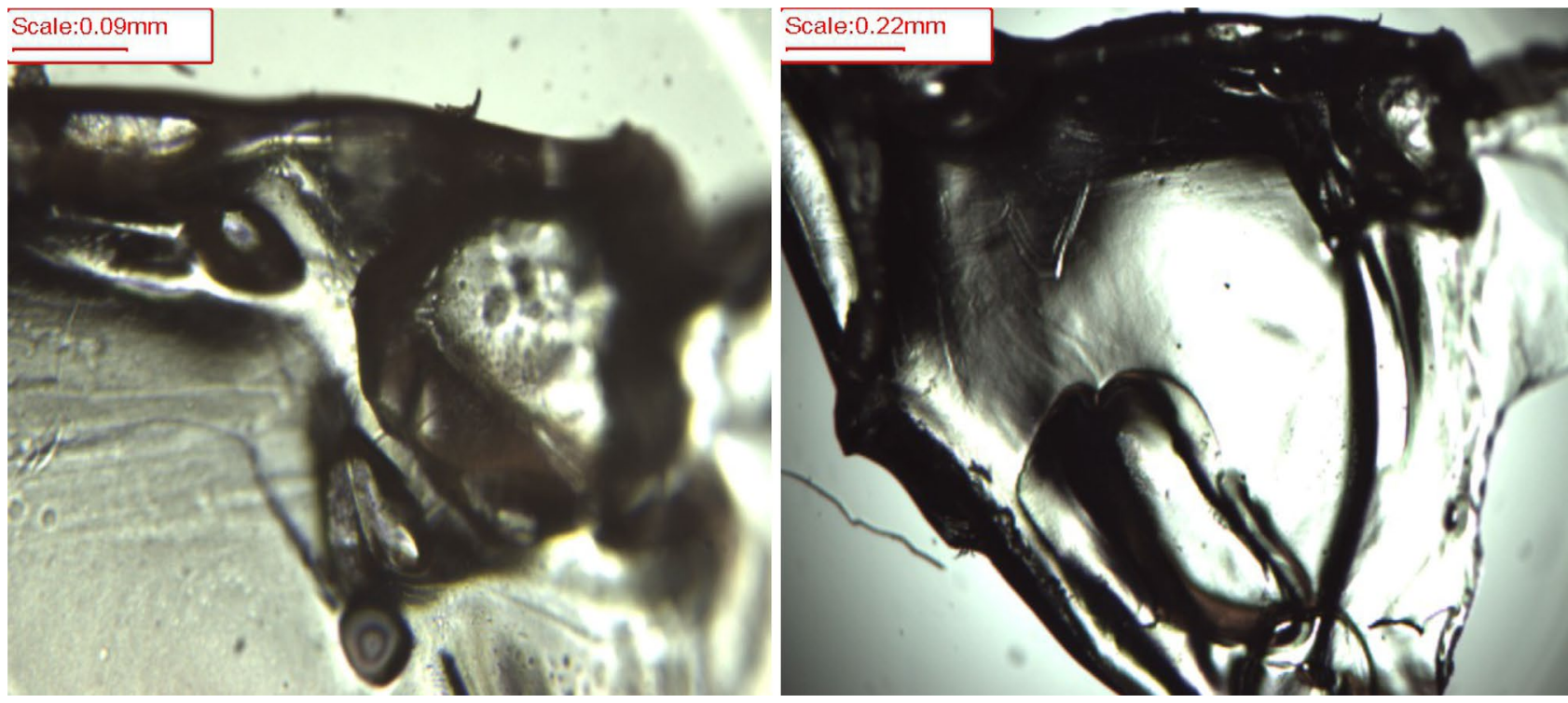

Fig. 5 Optical microscopic image of PVA/kefiran solutions with different magnification

had irregular diameter distributions and some very thick nanofibers were produced. Thus, $18 \mathrm{kV}$ was the optimum voltage for the production of kefiran/PVA composite nanofibers.

\subsection{Effect of tip-to-collector distance on nanofibers diameter}

One of the factors affecting the diameter of nanofibers and their morphology is the distance between the nozzle and collector. The nozzle-to-collector distance was changed from 120 to $200 \mathrm{~mm}$ in a concentration of $6 \%$ kefiran, a mixing ratio of $40 / 60 \mathrm{kefiran} / \mathrm{PVA}$ polymers, applied voltage of $18 \mathrm{kV}$, and the injection rate of $2 \mathrm{~mL} / \mathrm{h}$ SEM images; the diameter distributions of these nanofibers are shown in Fig. 3. And their mean diameter is shown in Table $1(\mathrm{j}-\mathrm{m})$. Figure 3 shows that in all conditions, soft, homogenous, and uniform nanofibers were produced. Moreover, the diameter of the nanofibers reduced as the distance increased.

\subsection{Effect of polymer injection rate on nanofiber diameter}

The effect of injection rate of polymer from nozzle to drum is shown in Fig. 4. Also, the mean diameters of nanofibers obtained from SEM images are shown in Table 1.

These images show that an increase in the mean diameter of the nanofibers occurred by increasing the injection rate. When the rate (polymer outlet speed) increased, the polymer jet had less time to be thrown from the nozzle to the drum as well as to dry up; thus, the diameter of the nanofibers increased. When the rate was the lowest, the polymer jet had enough time to be created; hence, thinner fibers with larger surface-to-volume ratio were produced.

\subsection{Preparation of optimum kefiran/PVA composite nanofibers}

By exegesis of SEM images, we can conclude that the optimum conditions of electrospinning parameters for the fabrication of kefiran/PVA nanofibers include the optimum voltage of $18 \mathrm{kV}$, the optimal distance of $200 \mathrm{~mm}$, and the injection rate of $1 \mathrm{~mL} / \mathrm{h}$ when the concentrations of kefiran and PVA are $6 \%$ and $8 \%$, respectively, with a mixing ratio

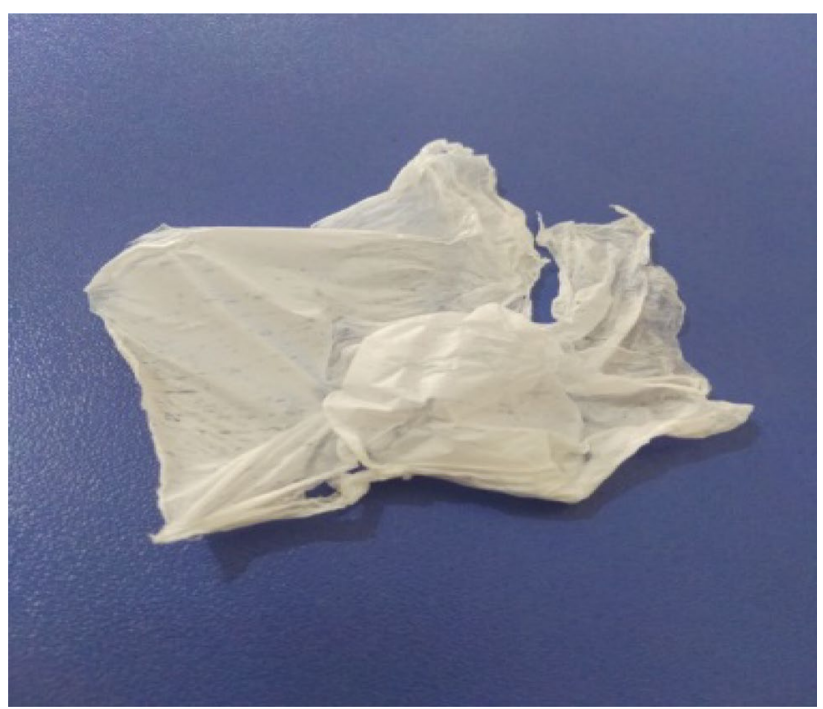

Fig. 6 Macroscopic image of kefiran/PVA nanofibers 

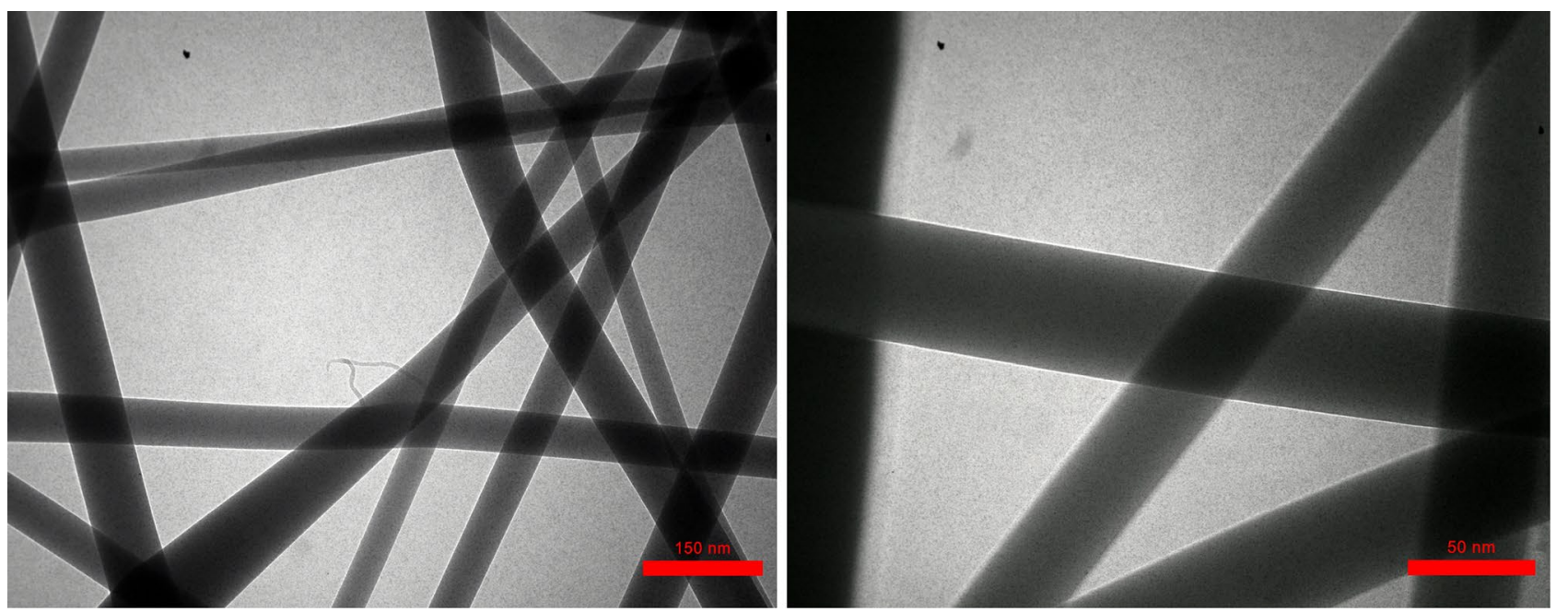

Fig. 7 Transmission electron microscopy (TEM) image of kefiran/PVA nanofibers with different magnification

of 40/60 w/w of kefiran/PVA polymers and the rotation of the drum in $375 \mathrm{rpm}$ rotary speed.

In this concentration, a homogenous solution was prepared with the addition of $4 \% \mathrm{w} / \mathrm{v}$ of Tween 80 . The homogeneity of PVA/kefiran blend solution was examined by optical microscope (Fig. 5). As is shown in Fig. 5, the $40 / 60 \mathrm{w} / \mathrm{w}$ of kefiran/PVA blend solution is fine, homogenous, and transparent without phase separation. On these conditions, the finest, homogenous, soft nanofibers with a mean diameter of $305 \mathrm{~nm}$ (Table 1 (n)) were fabricated. Not any knot or adhesion could be seen in the morphology of nanofibers. In addition, uniform distribution of nanofibers was observed. The nanofibers were collected for $4 \mathrm{~h}$ on foil and then removed from the foil. Figure 6 represents a macroscopic image of obtained nanofibers. As can be seen, it is stable, freestanding with a membrane-like appearance. The thickness of membrane was about $240 \pm 2$ micron. These nanostructures can be used as a coating on the food package, drugs, fabric, wound adhesive, carrier of medicine, patches, and filter. The transmission electron microscopy (TEM) image of kefiran/PVA composite nanofibers is shown in Fig. 7. The TEM image proves the homogeneity of nanofibers without any phase separation (Fig. 7).

\section{Conclusion}

The fabrications of kefiran/PVA composite nanofibers were carried out using the electrospinning method, and the effective parameters such as polymers mixing ratio, voltage, distance, and injection rate of the solution were changed to study their effects on the morphology and diameters of nanofibers. All conditions were characterized using SEM and size distributions, and diameters were calculated using Origin and Microsoft Measurement software. The results showed that the common solvent of PVA and kefiran was distilled water, so a blend solution was prepared by mixing PVA and kefiran polymers in water as a green solvent. Different proportions of polymers were examined for the electrospinning process. Adding kefiran solution to PVA solution up to $70 \% \mathrm{w} / \mathrm{w}$ was possible. Thus, increasing the temperature of the chamber makes it easy to produce kefiran/ PVA nanofibers through the electrospinning process. Electrospun kefiran/PVA composite nanofibers had an average diameter of about $305 \mathrm{~nm}$. Results showed that the diameter of kefiran/PVA nanofibers was higher than pure PVA nanofibers. The diameter of kefiran/PVA composite nanofibers increased as the content of kefiran in the spinning solution rose. Moreover, an increase in the applied voltage led to a decrease in the diameter of the nanofibers, and excessive overvoltage resulted in the formation of adhesion, knot creation, and a lack of uniform distribution of the diameters. Finally, it was observed that increasing the injection rate of polymer solution significantly increased the diameter of polyvinyl alcohol/kefiran nanofibers. The TEM image also proved that phase separation between two polymers did not occur.

Acknowledgements The authors would like to acknowledge the pharmaceutical Sciences Research Center, Tehran Medical Sciences, Islamic Azad University, for equipment and laboratory services. We would also like to express our gratitude to Dr. Farhad Tabandeh, University of Isfahan, for his help in proofreading the English language of the article. 


\section{Declarations}

Conflict of interest The authors declare that they have no conflict of interest.

\section{References}

1. Wang C, Wang J, Zeng L, Qiao Z, Liu X, Liu H, Zhang J, Ding $J$ (2019) Fabrication of electrospun polymer nanofibers with diverse morphologies. Molecules 24:834

2. Thenmozhi S, Dharmaraj N, Kadirvelu K, Yong Kim H (2017) Electrospun nanofibers: new generation materials for advanced applications. Mater Sci Eng, B 217:36-48

3. Kenry and Teck Lim C, (2017) Nanofiber technology: current status and emerging developments. Prog Polym Sci 70:1-17

4. Babitha S, Rachita L, Karthikeyan K, Shoba E, Janani I, Poornima B, PurnaSai K (2017) Electrospun protein nanofibers in healthcare: a review. Int J Pharm 523:52-90

5. Kamble P, Sadarani B, Majumdar A, Bhullar S (2017) Nanofiber based drug delivery systems for skin: a promising therapeutic approach. J Drug Deliv Sci Technol 41:124-133

6. Poláková L, Sirc J, Hobzová R, Cocarta AI, Heřmánková E (2019) Electrospun nanofibers for local anticancer therapy: review of in vivo activity. Int J Pharm 558:268-283

7. Massaglia G, Quaglio M (2018) emiconducting nanofibers in photoelectrochemistry. Mater Sci Semicond Process 73:13-21

8. Chou Sh, Carson D, Woodrow KA (2015) Current strategies for sustaining drug release from electrospun nanofibers. J Control Release 220:584-591

9. Hu X, Liu Sh, Zhou G, Huang Y, Xie Z, Jing X (2014) Electrospinning of polymeric nanofibers for drug delivery applications. J Control Release 185:12-21

10. Liang G, Yang L, Han Q, Chen G, Lin Ch, Chen Y, Luo L, Liu $\mathrm{X}, \mathrm{Li} \mathrm{Y}$ and Che $\mathrm{R}$ (2020) Conductive $\mathrm{Li}_{3.08} \mathrm{Cr}_{0.02} \mathrm{Si}_{0.09} \mathrm{~V}_{0.9} \mathrm{O}_{4}$ Anode material: novel "zero-strain" characteristic and superior electrochemical Li+ storage. Adv Energy Mater 1904267-1904278

11. Zhu X, Cao H, Li R, Fu Q, Liang G, Chen Y, Luo L, Lin Ch, Zhao XS (2019) Zinc niobate materials: crystal structures, energy storage capabilities and working mechanisms. J Mater Chem A 7:25537-25547

12. Lou X, Li R, Zhu X, Luo L, Chen Y, Lin Ch, Li H, Zhao XS (2019) New anode material for lithium-ion batteries: aluminium niobate $\left(\mathrm{AlNb}_{11} \mathrm{O}_{29}\right)$. ACS Appl Mater Interfaces 11:6089-6099

13. Suenaga S, Osada M (2019) Preparation of $\beta$-chitin nanofiber aerogels by lyophilization. Int J Biol Macromol 126:1145-1149

14. Yassin MA, Aziz MA, Ghanem AF, Abdel RM, H, (2019) Green synthesis of cellulose nanofibers using immobilized cellulose. Carbohyd Polym 205:255-260

15. Cui H, Bai M, Li C, Liu R, Lin L (2018) Fabrication of chitosan nanofibers containing tea tree oil liposomes against Salmonella spp. in chicken. LWT Food Sci Technol 96:671-678

16. Samadian H, Mobasheri H, Hasanpour S, Ai J, Azamie M, Faridi-Majidi R (2020) Electro-conductive carbon nanofibers as the promising interfacial biomaterials for bone tissue engineering. J Mol Liq 298:112021-112028

17. Hamida RS, Shami A, Ali MA, Almohawes ZN, Mohammed AE, Bin-Meferij MM (2021) Kefir: a protective dietary supplementation against viral infection. Biomed Pharmacother 133:110974-110985
18. Dhama K, Karthik K, Khandia R, Chakraborty S, Munjal A, Latheef SK et al (2018) Advances in designing and developing vaccines, drugs, and therapies to counter Ebola virus. Front Immunol 9:1803-1830

19. Ghasemlou M, Khodaiyan F, Jahanbin K, Gharibzahedi SMT, Taheri S (2012) Structural investigation and response surface optimisation for improvement of kefiran production yield from a low-cost culture medium. Food Chem 133:383-389

20. Exarhopoulos S, Raphaelides SN, Kontominas MG (2018) Conformational studies and molecular characterization of the polysaccharide kefiran. Food Hydrocolloids 77:347-356

21. Ghasemlou M, Khodaiyan F, Oromiehie A (2011) Physical, mechanical, barrier, and thermal properties of polyol-plasticized biodegradable edible film made from kefiran. Carbohyd Polym 84:477-483

22. Esnaasharian SS, Rezaeian S, Mirzaeian E, Afsharib H, Rezayat SM, Faridi-Majidi R (2014) Preparation and characterization of kefiran electrospun nanofibers. Int J Biol Macromol 70:50-56

23. Topuz F, Uyar T (2020) Antioxidant, antibacterial and antifungal electrospun nanofibers for food packaging applications. Food Res Int 130:108927-108931

24. Schmatz DA, Costa JAV, Morais MG, d, (2019) A novel nanocomposite for food packaging developed by electrospinning and electrospraying. Food Packaging and Shelf Life 20:100314-100322

25. Andre R. S, Mercante L. A, Farcure M. H. M, Payinatto A and Correa D. S (2021) Electrospun composite nanofibers as sensors for food analysis, Electrospun Polymers and Composites: Ultrafine Materials, High Performance Fibres and Wearables, Woodhead Publishing Series in Composites Science and Engineering, Pages 261-286

26. Senthil Muthu Kumar T, Senthil Kumar K, Rajini N, Siengchin S, Ayrilmis N, Varada Rajulu A (2019) A comprehensive review of electrospun nanofibers: food and packaging perpective. Compos B Eng 175:107074-107085

27. Torres-Giner S (2011). Electrospun nanofibers for food packaging applications. In J. M. Lagarón (Ed.), Multifunctional and Nanoreinforced Polymers for Food Packaging PP: 108125 Woodhead Publishing

28. Jenab A, Roghanian R, Emtiazi G, Ghaedi K (2017) Manufacturing and structural analysis of antimicrobial kefiran/poly ethylene oxide nanofibers for food packaging. Iran Polym J 26:31-39

29. Aslam M, Kalyar MA, Raza ZA (2018) Polyvinyl alcohol: a review of research status and use of polyvinyl alcohol based nanocomposites. Polym Eng Sci 58:2119-2132

30. Ben Halima N (2016) Poly (vinyl alcohol): review of its promising applications and insights into biodegradation. RSC Advances 6:39823-39832

31. Weiser D, Soti PL, Banoczi G, Bodai V, Kiss B, Gellert A, Nagy ZK, Koczka B, Szilagyi A, Marosi G, Poppe L (2016) Bioimprinted lipases in PVA nanofibers as efficient immobilized biocatalysts. Tetrahedron 72:7335-7342

32. Abdoli M, Sadrjavadi K, Arkan E, Zangeneh MM, Moradi S, Zangeneh A, Shahlaei M, Khaledian S (2020) Polyvinyl alcohol/Gum tragacanth/graphene oxide composite nanofiber for antibiotic delivery. J Drug Deliv Sci Technol 60:102044

33. Qiu H, Zhu S, Pang L, Ma J, Liu Y, Du L, Wu Y, Jin Y (2020) ICG-loaded photodynamic chitosan/polyvinyl alcohol composite nanofibers: anti-resistant bacterial effect and improved healing of infected wounds. Int J Pharm 588:119797

34. Frone AN, Panaitescu DM, Donescu D, Spataru CI, Radovici C, Trusca R, Somoghi R (2011) Preparation and characterization of PVA composites with cellulose nanofibers obtained by ultrasonication. Bio Resources 6:487-512 
35. Zhang X, Tang K, Zheng X (2016) Electrospining and crosslinking of COL/PVA nanofiber-microsphere containing salicylic acid for drug delivery. J Bionic Eng 13:143-149

36. He M, Zhang B, Dou Y, Yin G, Cuia Y, Chen X (2017) Fabrication and characterization of electrospun feather keratin/poly(vinyl alcohol) composite nanofibers. RSC Advances 7:9854-9861

37. Yang X, Guo Y, Han Y, Li Y, Ma T, Chen M, Kong J, Zhu J, Gu J (2019) Significant improvement of thermal conductivities for BNNS/PVA composite films via electrospinning followed by hot-pressing technology. Compos B 175:107070

38. Shahabi-Ghahfarrokhi I, Khodaiyan F, Mousavi M, Yousefi H (2015) Green bionanocomposite based on kefiran and cellulose nanocrystals produced from beer industrial residues. Int J Biol Macromol 77:85-91
39. Bagherian far M and Ziyadi H, (2016) Fabrication of polyvinyl alcohol/kefiran nanofibers membrane using electrospinning. J Pharm Health Sci 4:211-218

40. Jiang T, Carbone EJ, Lo KWH, Laurencin CT (2015) Electrospinning of polymer nanofibers for tissue regeneration. Prog Polym Sci 46:1-24

41. Haider A, Haider S, Kang IA (2018) comprehensive review summarizing the effect of electrospinning parameters and potential applications of nanofibers in biomedical and biotechnology. Arab J Chem 11:1165-1188

Publisher's Note Springer Nature remains neutral with regard to jurisdictional claims in published maps and institutional affiliations. 